\title{
Protograph LDPC Code Design for LS-MIMO 1-bit ADC Systems
}

\author{
Hung N. Dang, Thuy V. Nguyen \\ Vietnam \\ Correspondence: Thuy V. Nguyen, thuynv@ptit.edu.vn \\ Communication: received 9 March 2021, revised 17 April 2021, accepted 26 April 2021 \\ Online publication: 4 June 2021, Digital Object Identifier: 10.21553/rev-jec.266 \\ The associate editor coordinating the review of this article and recommending it for publication was Prof. Vo Nguyen Quoc Bao.
}

Faculty of Information Technology, Posts and Telecommunications Institute of Technology, Hanoi,

\begin{abstract}
Recently, two emerging research topics are protograph low-density parity-check (P-LDPC) and large-scale multiinput multi-output (LS-MIMO) with low-resolution analog-to-digital (ADC) converters (LS-MIMO-LOW-ADC). In these directions, many research works have proposed 1-bit ADC as a good candidate for LS-MIMO systems in order to save both transmission power and circuit energy dissipation. However, we observed that previously reported P-LDPC codes might not have good performance for LS-MIMO systems with 1-bit ADC. Hence, we perform a re-design of the P-LDPC codes for the above systems in this paper. The new codes demonstrate a good coding gain from $0.3 \mathrm{~dB}$ at rate $1 / 2$ to $0.5 \mathrm{~dB}$ at rate 2/3 in different LS-MIMO configurations with 1-bit ADC.
\end{abstract}

Keywords- 1-bit ADC, Protograph LDPC Code, Large-Scale MIMO, PEXIT

\section{INTRODUCTION}

In the future generation wireless networks $(5 \mathrm{G}, 6 \mathrm{G})$, LDPC codes and LS-MIMO are two promising technologies [1, 2]. In particular, the LDPC codes stand out with practical advantages such as low complexity decoder / encoder structure, capacity approaching error correction performance [3-6]. The LS-MIMO systems with a large number of transmitting/receive antennas have many advantages such as increased spectral efficiency, data rates, and reliability, respectively, without the need for extra power and bandwidth. However, the LS-MIMO systems pose several technical challenges to the radio frequency (RF) module on the receiver side. Analog to digital converters (ADCs) are the components that consume the majority amount of the power, and their hardware costs are also high. The hardware cost and power consumption of ADCs increase exponentially with the ADC resolution. One viable solution to deal with these challenges is to replace highresolution ADCs with low-resolution ADCs [7-15].

One of the problems with low-resolution ADCs is a performance loss due to hardware defects. Xu et al. [12] shown that increasing the number of receiving antennas might help to reduce this performance degradation. However, another challenge is that low-resolution ADCs cause floor error behavior for the channel estimator. To solve this problem, Nguyen et al. [11], and Gao et al. [10] used a method of [12] that combined with deep learning techniques to solve the channel estimation problem, but for large MIMO with mixed ADC. For the case of 1-bit ADC, where message passing channel decoder is used, Shao et al. [16], Cho et al. [14] introduced various algorithms to calculate the loglikelihood ratio (LLR) for the MIMO signal detector.
In this approach, the LLR values can be exchanged between the MIMO detector and the message-passing channel decoder to improve the performance with iterative algorithms.

Regarding past works related to LS-MIMO systems with low-resolution ADC module [10, 13, 15], we found that there is a limited number of previous research works that study the performance of the LDPC codes in these LS-MIMO systems where 1-bit ADC exploited. Recently, a related study by Vu et al. [13] had proposed a method to analyze and evaluate a joint graph of P-LDPC and LS-MIMO by exploiting the protograph based extrinsic information exchange chart (PEXIT) for a joint scheme of protograph LDPC decoder and the belief propagation massive MIMO detection. The study has shown the significant gain of a joint iterative MIMO detection and LDPC decoding scheme compared with past coded LS-MIMO detection methods. Then, Dang et al. [17], extended the method of $\mathrm{Vu}$ et al. [13], investigated the new optimized quantizer for low-bit ADC, e.g., 2-bit to 5-bit ADCs, which showed better performance over classical 3-sigma rule quantization in the traditional ADC methodology. Furthermore, the experiment results also indicated that the new quantizer provided a significant performance improvement for LS-MIMO 1-bit ADC system [18]. This work also showed the poor performance at a higher coding rate $R=2 / 3$ and the error floor at $\mathrm{BER}=10^{-2}$ with the 1-bit ADC $10 \times 10 \mathrm{MIMO}$ configuration.

The above issue might be due to the reported PLDPC codes that were not optimized for 1-bit ADC LS-MIMO systems. For that reason, this paper designs new protograph LDPC codes for the LS-MIMO coded systems with 1-bit ADC. In our proposed design, both the iterative decoding threshold of protograph codes 


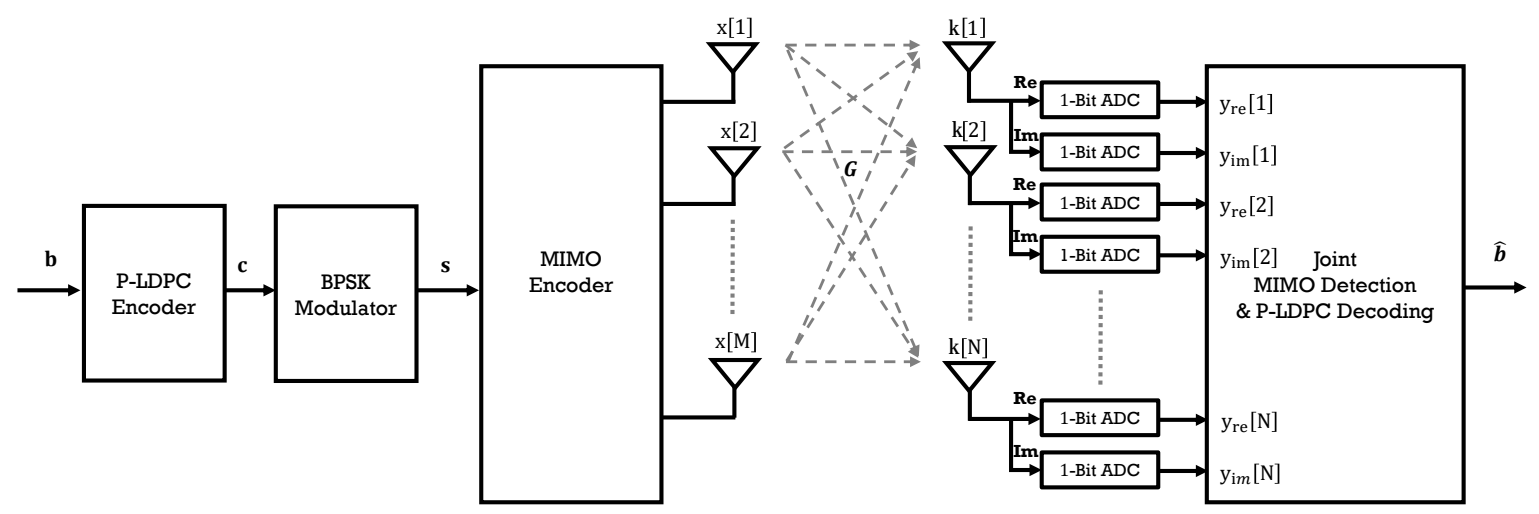

Figure 1. The channel model of the LS-MIMO coded communication with 1-bit ADC.

and the frame error rate (FER) will be taken into consideration. The main contributions of the paper are summarized in the following:

- We propose a new design of high-performing PLDPC codes suitable for LS-MIMO systems with 1-bit ADC. The new coding scheme overcomes the error floor issue of previously reported LDPC codes. The new coded LS-MIMO systems have error-floor behavior at FER as low as $10^{-4}$. Such performance achievement is promising for the new generation wireless networks, where reliability is one of the top priorities.

- The investigation in this research reveals that the new LDPC codes, optimized for 1-bit ADC, achieve better performance than all chosen LDPC codes in $M / N=1$ MIMO configuration.

- Observing the system's performance at $M / N<1$ MIMO configuration suggests that the new LSMIMO-PEXIT algorithm should be re-optimized for this particular case to achieve better system performance.

The remaining paper is organized as follows. Section 2 presents the system model for LS-MIMO 1bit ADC channel and the receiver's joint double-layer belief propagation algorithm. In Section 3, an improved version of the PEXIT algorithm is proposed and used as the main component in the design framework of protograph LDPC codes. Besides, we utilize a two-step procedure to search for new protograph LDPC codes that do not have the error-floor behavior at the FER level as low as $10^{-4}$. The proto-matrices of the new protograph LDPC codes optimized for a maximum number of decoding iterations are 50 and $10 \times 10$ MIMO configurations, are presented in Section 4 . Here, the protograph LDPC codes for massive MIMO channel [19], and the new optimized protograph LDPC codes are chosen to perform FER performance analysis. Finally, Section 5 concludes the paper.

\section{System Model}

\subsection{Channel Model}

Consider a wireless fading multiple-input-multipleoutput (MIMO) channel with $M, N$ transmitting, receiv- ing antennas, respectively. In the model, the receiver exploits 1-bit ADCs at the output of each receive antenna, as shown in Figure 1.

First, the P-LDPC encoder takes an input block of $L_{i}$ information bits, and produces a codeword with a length of $L_{c}$ coded bits $c \in\{0,1\}$. The coding rate $R$ is determined as $R=L_{i} / L_{c}$. Next, the coded bits are modulated by a binary-phase-shift-keying (BPSK) modulator $s=(-1)^{c} \in\{+1,-1\}$ and transmit to channel using $M$ antennas using the spatial multiplexing scheme [20]. To transmit all of $L_{c}$ coded bits, the system exploits $L=\left\lceil L_{c} / M\right\rceil$ channel uses.

The massive MIMO channel is modeled in the following

$$
\mathbf{k}=\mathbf{G} \mathbf{x}+\mathbf{w}_{\mathbf{a}},
$$

where $\mathbf{x}=[x[1], x[2], x[3], \ldots, x[M]]^{T}$ is the transmitted MIMO symbol. $\mathbf{G} \in \mathbb{C}^{N \times M}$ is channel gain matrix whose entries $g[n, m]$ in the $n$-th row and $m$-th column of $\mathbf{G}$ are assumed to be i.i.d complex Gaussian with zero mean and unit variance $\mathcal{C N}(0,1)$. The average symbol energy $E_{s}=\mathbb{E}\left(\|\mathbf{x}\|^{2}\right)$ is normalized to 1 . Finally, $\mathbf{k}=[k[1], k[2], k[3], \ldots, k[N]]^{T} \in \mathbb{C}^{N \times 1}$ is the received signal vector with each element $k[n]$ is the received signal at the $n$-th antenna.

In the scope of this paper, we assume that the perfect channel state information (CSI) is available at the receiver only. The noise vector $\mathbf{w}_{\mathbf{a}}=$ $\left[w_{a}[1], w_{a}[2], w_{a}[3], \ldots, w_{a}[N]\right]^{T} \in \mathbb{C}^{N \times 1}$ is assumed to be complex additive white Gaussian noise vector. In this vector, each element follow i.i.d complex Gaussian with zero mean and $N_{0}$ variance (i.e., $\mathcal{C N}\left(0, N_{0}\right)$ ).

As shown in Figure 1, at the output of each receiving antenna, a pair of 1-bit ADC is exploited to transform the received signals, $k[n], n=1,2,3, \ldots, N$ from analog form to digital bits. One 1-bit ADC applies for the in-phase (real) signal, and the other 1-bit ADC is for the quadrature (imaginary). Finally, to restore the original information bits, the quantized version of the received signal is fed to the joint MIMO detection and protograph LDPC decoding algorithm [13]. In the next sections, we will summarize the 1-bit quantization model and the joint MIMO detection and protograph LDPC decoding algorithm. 


\subsection{1-Bit Quantization Modeling}

The relationship between the input and output of the 1-bit ADC block is given by

$$
\mathbf{y}=\mathbb{Q}\left(\mathbf{k}_{r e}\right)+j \mathbb{Q}\left(\mathbf{k}_{i m}\right),
$$

where $\mathbf{Q}$ is denoted as the quantization operator, $\mathbf{k}_{r e}$ and $\mathbf{k}_{i m}$ are the real and imaginary parts of the received signal k, respectively. Note that $\mathbb{Q}$ is the scalar and uniform quantizer.

In this work, we exploit the additive quantization noise model (AQNM) in MIMO systems with lowresolution ADCs [21, 22] to optimize the 1-bit ADC model. Here, the quantization noise is modeled as the noise component that is added to the input signal. Thus (2) can be rewritten as [21]

$$
\mathbf{y}=\varphi \mathbf{k}+\mathbf{w}_{Q},
$$

where $\mathbf{w}_{Q}$ is the additive quantization noise and $\varphi=$ $1-\rho$, which is the performance metric of a given quantizer, with $\rho$ is the inverse of the signal-to-quantizationdistortion ratio.

Noted that the larger the value of $\varphi$ is, the better the performance of the massive MIMO coded communication system [18]. Thus, to improve the performance of a given massive MIMO coded communication system, one can seek a higher value of $\varphi$ for a given 1-bit ADC by reducing the quantization noise of the quantizer. In recent works [17, 18], Dang et al. showed that by optimizing the quantizer's truncation limit, the quantization noise would dramatically be reduced. More specifically, at 1-bit ADC or only two quantization levels, the value $\varphi$ of the optimized quantizer is 0.6261 , which is more than 0.489 when compared with the value $\varphi$ of the 3 -sigma quantizer, which is $0.1371^{1}$. Also, the $\varphi$ value of the optimized quantizer is also very close to the non-uniform quantizer, about 0.01 , a marginal gap. This proves that this optimized linear quantizer would have a performance close to a nonlinear one. The close performance gaps were verified via both the iterative decoding threshold and numerical simulations under the various experiment of the MIMO configurations and code rates in [18].

\subsection{Joint MIMO Detection and Protograph LDPC Decoding}

The joint MIMO detection and protograph LDPC decoding algorithm, [13, 19], is a promising solution for the massive MIMO coded communication systems because of two main reasons:

1) Excellent performance since the receiver allows the extrinsic information exchanged iteratively between the MIMO detector layer and the protograph LDPC code layer.

2) Low complexity receiver [23].

In the following, we also apply this joint detection and LDPC architecture for our code design that is best suited for the 1-bit ADC LS-MIMO systems. It is worth

\footnotetext{
${ }^{1}$ This also explains why the 3-sigma quantizer has a poor performance in 1-bit ADC LS-MIMO systems
}

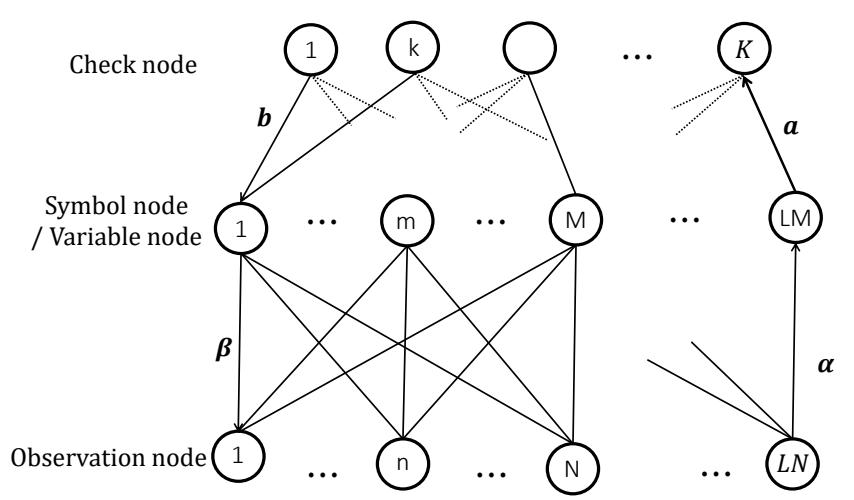

Figure 2. Joint MIMO detection and protograph LDPC decoding.

noting that LS-MIMO systems employ a high number of antennas, in order of tens or hundreds. Thus the traditional MIMO detection algorithms such as zeroforcing, minimum mean square error spatial or sphere decoding, are not applicable due to computational restriction $[23,24]$.

Moreover, Nguyen et al. [13] and Vu et al. [19] employed similar ideas in the joint belief propagation decoder for large-scale MIMO coded communication systems to analyze the performance of low-resolution ADCs (from 2-bit to 5-bit).

Even though these research works were related to the topic addressed in this paper. But, neither of them deals with the performance of the coded LS-MIMO systems with 1-bit ADCs. The recent work [18] was filled the gap to investigate and present the performance of 1-bit ADCs with the joint MIMO detection and protograph LDPC decoding. To continue this work, the new protograph LDPC codes should be redesigned for the 1-bit ADC case, which is the main contribution of our paper.

The joint detection and decoding algorithm can be represented by a double-layer graph, as shown in Figure 2. In that structure, there are three types of nodes:

1) $L \times N$ observation nodes are the received signals $\mathbf{y}$.

2) $L_{c}=L \times M$ symbol/variable nodes are the transmit symbol sequence $\mathbf{x}$.

3) Finally, there are $K=L_{c}-L_{i}$ check nodes of the given P-LDPC codes.

In one channel use, the $N$ observation nodes and the $M$ symbol nodes are fully connected to create a full graph for the MIMO detection part (i.e., one observation node is connected to all $M$ symbol nodes via the wireless channel). In the LDPC decoding graph part, the parity matrix of the LDPC code defines the connection of the check node and the variable node. Here, there are $L_{c}$ variable nodes representing the sequence $\mathbf{c}$. With the BPSK modulation scheme, the codeword bit and the transmitting symbol are mapped one-one. That is why in Figure 2, the variable node and the symbol node are joined in a single node on the double-layer graph.

In the iterative joint detection and decoding algorithm on the double-layer graph, five types of messages are passed over the graph, $\alpha, \mathbf{a}, \mathbf{b}, \beta, \gamma$, which are 
calculated [13] by the following formulas as belows:

- $\alpha[n, m]$ is the message passed from the $n$-th observation node to the $m$-th symbol node

$$
\begin{aligned}
\alpha[n, m] & =\ln \frac{\operatorname{Pr}(\hat{y}[n, m] \mid \mathbf{G}, x[m]=+1)}{\operatorname{Pr}(\hat{y}[n, m] \mid \mathbf{G}, x[m]=-1)} \\
& =\frac{4 \varphi}{\Psi[n, m]} \mathfrak{R}\left(g^{*}[n, m] \hat{y}[n, m]\right) .
\end{aligned}
$$

where

$$
\begin{aligned}
\Psi[n, m] & =\varphi^{2} \sum_{t=1, t \neq m}^{M}|g[n, t]|^{2}\left(1-|\hat{x}[n, t]|^{2}\right) \\
& +\varphi^{2} N_{0}+\varphi(1-\varphi)\left(\sum_{m=1}^{M}|g[n, m]|^{2}+N_{0}\right) .
\end{aligned}
$$

- $\mathbf{a}[m, k]$ is the message passed from the $m$-th variable node to the $k$-th check node

$$
\mathbf{a}[m, k]=\sum_{t \in \mathcal{N}_{o}(m)} \alpha[t, m]+\sum_{t \in \mathcal{N}_{c}(m) \backslash k} \mathbf{b}[t, m],
$$

- $\mathbf{b}[k, m]$ is the message passed from the $k$-th check node to the $m$-th variable node

$$
\mathbf{b}[k, m]=\ln \frac{1-\prod_{t \in \mathcal{N}_{v}(k) \backslash m} \frac{1-e^{a[t, k]}}{1+e^{a[t, k]}}}{1+\prod_{t \in \mathcal{N}_{v}(k) \backslash m} \frac{1-e^{a[t, k]}}{1+e^{a[t, k]}},}
$$

- $\beta[m, n]$ is the message passing from the $m$-th symbol node to the $n$-th observation node

$$
\beta[m, n]=\sum_{t \in \mathcal{N}_{o}(m) \backslash n} \alpha[t, m]+\sum_{t \in \mathcal{N}_{c}(m)} \mathbf{b}[t, m],
$$

- $\gamma[m]$ is the a posteriori log-likelihood ratio value of the symbol $x[m]$

$$
\hat{c}[m]= \begin{cases}0, & \gamma[m]>0 \\ 1, & \text { Otherwise. }\end{cases}
$$

where $\hat{c}[m]$ denotes the decoded version of $c[m]$. And thus, the decoded sequence of the information $\hat{\mathbf{b}}$ is obtained.

The message-passing process stops when all check equations are satisfied or the maximum number of iterations is reached. Otherwise, the message-passing process repeats with a message update from the observation nodes.

Based on the double-layer graph, the protograph extrinsic information chart algorithm was previously derived in [13]. This algorithm will be used to optimize the protograph LDPC code for 1-bit ADC in the below section.

\section{Code Design}

The essence of the protograph LDPC code design is the search for a protomatrix $\mathbf{P}$ that satisfies the lowest iterative decoding threshold while maintaining the linear minimum distance growth property. This can be presented as an optimization problem as follows [11]:

$$
\begin{aligned}
\min _{\mathbf{P} \in \mathcal{P}_{E^{+}}} & \xi\left(\mathbf{P}, N, M, \text { Iter }_{\max }\right) \\
\text { s.t. } & f_{r}(\mathbf{P}) \leq 0 \text { for } r=1,2, \ldots, R,
\end{aligned}
$$

where $\mathcal{P}_{E^{+}}$denotes a set of all matrices with non-negative entries, the output of cost function $\xi(\mathbf{P}, M, N$, Iter $\max )$ is the iterative decoding threshold value. Note that the cost function is obtained by applying the LS-MIMO-PEXIT algorithm in Section 2. Finally, $f_{r}(\mathbf{P}) \leq 0, r=1,2, \ldots, R$ are the set of constraints according the design guidelines of protograph LPDC codes [25].

In this work, we will use three main parameters involved in the optimization problem: 1) A pair of $M$ and $N$ represents the LS-MIMO configuration; 2) Protomatrix $\mathbf{P}$ represents the code structure of a given coding rate; 3 ) Iter $_{\max }$ represents the maximum number of decoding iterations which is often limited due to the latency constraint of a given wireless communication system. Unlike previous existing protograph code designs, e.g., $[25,26]$, in the optimization problem (10) above, we take Iter $_{\max }$ as a design parameter to optimize the protograph LDPC code for 1-bit ADC case. For that, we optimize the performance on both waterfall and error-floor areas.

Using guidelines on the properties of a good protomatrix [25], we can limit the search space of the above optimization problem. This allowed us to perform the brute-force search with reasonable complexity. To start, we set constraints on the structure of the beginning proto matrix $\mathbf{P}$, at a coding rate of $1 / 2$.

$$
\mathbf{P}_{1 / 2}=\left(\begin{array}{llllll}
e_{1,1} & e_{1,2} & e_{1,3} & e_{1,4} & 0 & 1 \\
e_{2,1} & e_{2,2} & e_{2,3} & e_{2,4} & 1 & 0 \\
e_{3,1} & e_{3,2} & e_{3,3} & e_{3,4} & 1 & 0
\end{array}\right)_{3 \times 6},
$$

where element $e_{i, j}$ in $\mathbf{P}_{1 / 2}$ is the number of parallel edges connecting the $s^{\text {th }}$ check node and the $p^{\text {th }}$ variable node in the LDPC decoding part of the joint protograph. The last two columns are pre-selected according to the design guideline on the number of degree-one variable nodes and degree-2 variable nodes [25].

The remaining four columns have a total of 12 search variables $e_{i, j}, i=1,2,3, j=1, \ldots, 4$ to optimize. The maximum number of allowed parallel edges is 3 (i.e. Those variable values are selected from the set $\{0,1,2,3\})$. Finally, the corresponding constraints $f_{r}(\mathbf{P})$ of the optimization problem in (10) for the above matrix $\mathbf{P}_{1 / 2}$ are as follows [25]:

$$
\left\{\begin{array}{l}
f_{1}\left(\mathbf{P}_{1 / 2}\right): e_{i, j} \geq 0, \forall s=1,2,3, p=1, \cdots, 4 \\
f_{2}\left(\mathbf{P}_{1 / 2}\right): e_{i, j} \leq 3, \forall s=1,2,3, p=1, \cdots, 4 \\
f_{3}\left(\mathbf{P}_{1 / 2}\right):\left(e_{1,1}+e_{2,1}+e_{3,1}\right) \leq 3 \\
f_{4}\left(\mathbf{P}_{1 / 2}\right):\left(e_{1,2}+e_{2,2}+e_{3,2}\right) \leq 3 \\
f_{5}\left(\mathbf{P}_{1 / 2}\right):\left(e_{1,3}+e_{2,3}+e_{3,3}\right) \leq 3 \\
f_{6}\left(\mathbf{P}_{1 / 2}\right):\left(e_{1,4}+e_{2,4}+e_{3,4}\right) \leq 2
\end{array}\right.
$$

The constraints $f_{3}\left(\mathbf{P}_{1 / 2}\right), f_{4}\left(\mathbf{P}_{1 / 2}\right), f_{5}\left(\mathbf{P}_{1 / 2}\right)$ are imposed to guarantee the linear minimum distance growth, and the constraint $f_{6}\left(\mathbf{P}_{1 / 2}\right)$ comes from the fact that a good protograph LPDC code can have up to the number of check nodes minus 1 or $(3-1=2)$ degree- 2 variable nodes allowed in the final protograph [27]. It is noted that the constraint on the number of degree-2 
variable nodes in the proto-matrix is the necessary, but not the sufficient condition for a good LDPC code. However, imposing this constraint to narrow down the search space is a good practice for AWGN channels in the literature, $[25,26]$. We performed an experiment for LS-MIMO channels by searching for a best protomatrix on the full search space (i.e., we impose only two constraints $f_{1}\left(\mathbf{P}_{1 / 2}\right):-e_{i, j} \leq 0$ and $\left.f_{2}\left(\mathbf{P}_{1 / 2}\right): e_{i, j}-2 \leq 0\right)$. The experiment result reveals that the best proto-matrix follows the guideline above. That is, the best protomatrix has a maximum of two degree- 2 variables, one degree-1 variable, and all remaining variables have degrees higher than 3 . Therefore, we employ the guideline to design new protograph LDPC codes for 1-bit ADC LS-MIMO systems in this paper.

\section{EXPERIMENT Results ANd Discussions}

First of all, we carry out the code search with Iter $_{\max }=$ 50 and $M=10, N=10$ (i.e., $10 \times 10$ LS-MIMO configuration). We are interested in Iter $_{\max }=50$, the number of decoding iterations because the low latency is one of the critical requirements in the future wireless communications $^{2}[1,2]$, but it should be high enough to deal with the processing power limitation of 1-bit ADC. The optimal protomatrices for the coding rate of $1 / 2,2 / 3$ is given below:

$$
\begin{gathered}
\mathbf{P}_{1 / 2}^{50 \text { iter. }}=\left(\begin{array}{llllll}
3 & 2 & 0 & 0 & 0 & 1 \\
2 & 2 & 1 & 1 & 1 & 0 \\
2 & 1 & 2 & 1 & 1 & 0
\end{array}\right)_{3 \times 6}, \\
\mathbf{P}_{2 / 3}^{50 \text { iter. }}=\left(\begin{array}{lllllllll}
2 & 0 & 2 & 3 & 2 & 0 & 0 & 0 & 1 \\
0 & 1 & 2 & 2 & 2 & 1 & 1 & 1 & 0 \\
0 & 2 & 1 & 2 & 1 & 2 & 1 & 1 & 0
\end{array}\right)_{3 \times 9}
\end{gathered}
$$

It should be noted that those proto-matrices in (13), (14) are not the ones that have the lowest iterative decoding thresholds. Observation from our practical design experience that the proto-matrix with the lowest iterative decoding threshold sometimes has the error-floor behavior. Hence, a search will be performed starting from the lowest iterative decoding threshold, increasing gradually until the best value of the desired threshold is reached. Incorporating the practical design experience, we introduce a two-step procedure to optimize the protograph LDPC codes as below [11]:

- Step 1: The coarse step where we choose a set of new proto-matrix into a buffer if its iterative decoding threshold, using the LS-MIMO-PEXIT algorithm, is lower than the expected threshold. Consequently, when the coarse step finishes, we obtain a list of ten proto-matrices the lowest thresholds.

- Step 2: In the filtration step, we perform intensive simulations to filter out the error-floor protomatrices. Finally, we choose the proto-matrix that

\footnotetext{
${ }^{2}$ It is worth noting that Iter $_{\text {max }}$ indicates the maximum number of iterations that the receiver can tolerate if there is an error. However, the receiver will stop as soon as a valid codeword is found.
}

achieves FER $=10^{-4}$ at the lowest SNR from the error-floor-free list at the output of the filter.

To illustrate the advantage of the two-step design procedure mentioned above, using the channel model at Section 2.1, FER performance of rate 1/2 protograph LDPC code in (13) are shown in Figures 3-6. One can see that the reported protograph LDPC code does not have error-floor behavior at the FER $=10^{-4}$. This attribute of the proposed codes makes them useful for the new generation of wireless networks where ultrareliability is often required.

Next, we pick two protograph LDPC codes from the literature to compare with our new codes - AR3A codes [28] (punctured codes) and the protograph LDPC codes previously optimized for LS-MIMO channels and joint double-layer belief propagation receiver [19] (NND codes). The reason behind this choice is that the AR3A codes have three checks, the same as the number of checks of our proposed codes. The AR3A codes were reported to achieve the best performance in the fading environment [28]. Also, the NND codes [19] are chosen since they are the only codes, to our best understanding, that satisfies two conditions: 1) belong to non-punctured class; 2) have the same check and were optimized for low-resolution ADC with a fixed number of decoding iterations. Those two conditions are required to achieve a fair comparison with our proposed codes.

As seen in Figure 3 and Figure 4, the proposed codes outperform all other codes $[19,28]$ with a coding gain of from 0.3 to $0.7 \mathrm{~dB}$ at FER $=10^{-4}$ for the code rate of $1 / 2$. At the higher code rate, $2 / 3$, the coding gains in comparison with the NND code and AR3A code are about from $0.5 \mathrm{~dB}$ and $1.0 \mathrm{~dB}$, respectively. The same is seen as the coding gain of the $10 \times 10$ and $100 \times 100$ MIMO configuration. This investigation means that the performance of our proposed protograph LDPC codes is sensitive to the $M / N=1 \mathrm{MIMO}$ configurations.

Another direction, the FER performance gap vanishes at the $10 \times 40$ and $10 \times 100 \mathrm{MIMO}$ configuration as shown in Figure 5 and Figure 6. One can see that the performance of the proposed codes is very close to the AR3A codes and NND codes in both rates $1 / 2$ and $2 / 3$. The observation from this investigation means that the performance gain of our proposed protograph LDPC codes is marginal with the $M / N<1$ MIMO configurations, and there will be our next research topics shortly.

It should be noted that when the number of the receiving antennas is extremely higher than the number of transmit antennas, the previously AWGN-optimized code, AR3A, delivers the best performance in the 1-bit ADC LS-MIMO communication systems.

\section{Conclusion And Future Works}

This paper re-design the protograph LDPC codes for 1bit ADC LS-MIMO systems. The new protograph codes yield the coding gains from $0.3 \mathrm{~dB}$ to $0.7 \mathrm{~dB}$ over the offthe-shelf protograph LDPC codes. These coding gains 


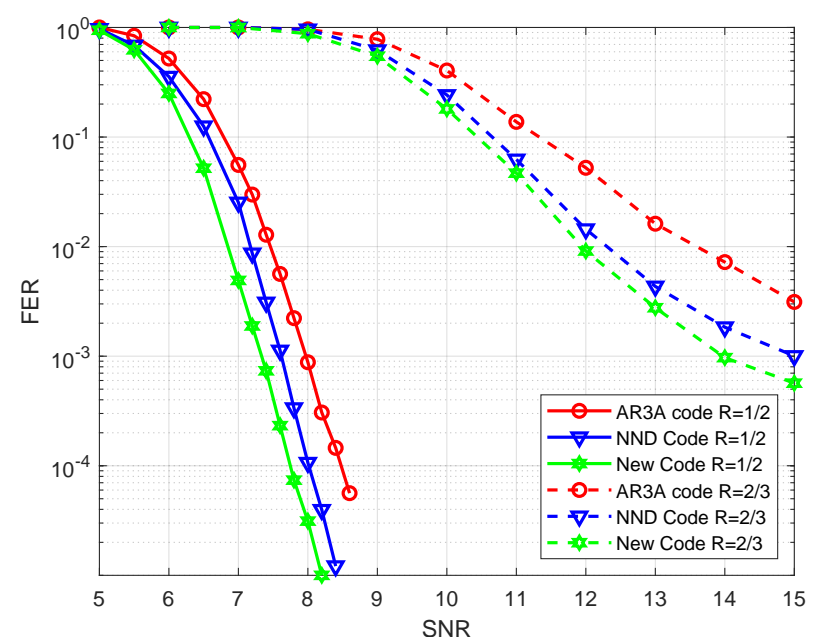

Figure 3. FER performance: Coding rate $R=1 / 2$ and $R=2 / 3$, information blocklength 2400 bits, 50 iterations, $10 \times 10$ LS-MIMO.

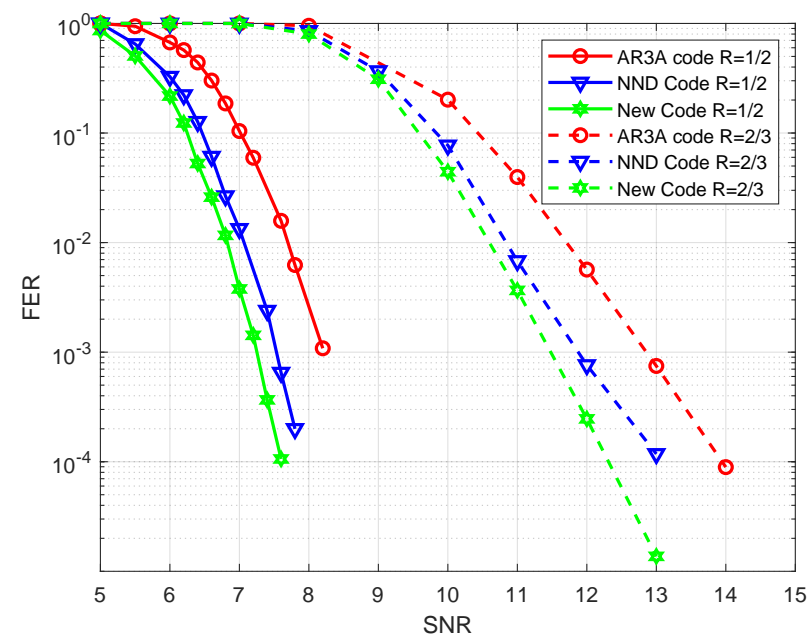

Figure 4. FER performance: Coding rate $R=1 / 2$ and $R=2 / 3$, information blocklength 2400 bits, 50 iterations, $100 \times 100$ LS-MIMO.

are significant, especially for the high-speed wireless communications system where the battery-operated devices' power supply is strictly limited.

\section{ACKNOWLEDGMENT}

Hung N. Dang was funded by Vingroup Joint Stock Company and supported by the Domestic Ph.D. Scholarship Programme of Vingroup Innovation Foundation (VINIF), Vingroup Big Data Institute (VINBIGDATA), code VINIF.2020.TS.130.

\section{REFERENCES}

[1] Z. Zhang, Y. Xiao, Z. Ma, M. Xiao, Z. Ding, X. Lei, G. K. Karagiannidis, and P. Fan, "6G wireless networks: Vision, requirements, architecture, and key technologies," IEEE Vehicular Technology Magazine, vol. 14, no. 3, pp. 28-41, Sep. 2019.

[2] K. B. Letaief, W. Chen, Y. Shi, J. Zhang, and Y. A. Zhang, "The roadmap to 6G: AI empowered wireless networks,"

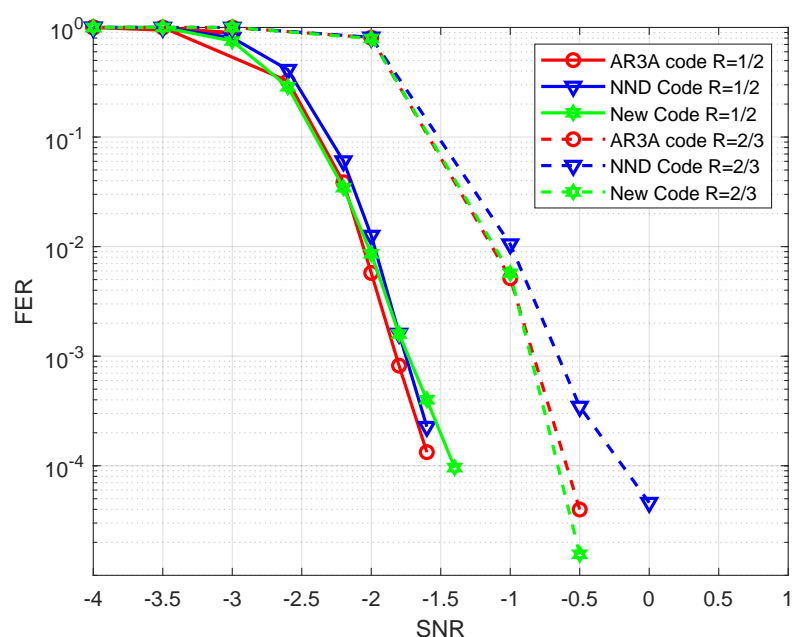

Figure 5. FER performance: Coding rate $R=1 / 2$ and $R=2 / 3$, information blocklength 2400 bits, 50 iterations, $10 \times 40$ LS-MIMO.

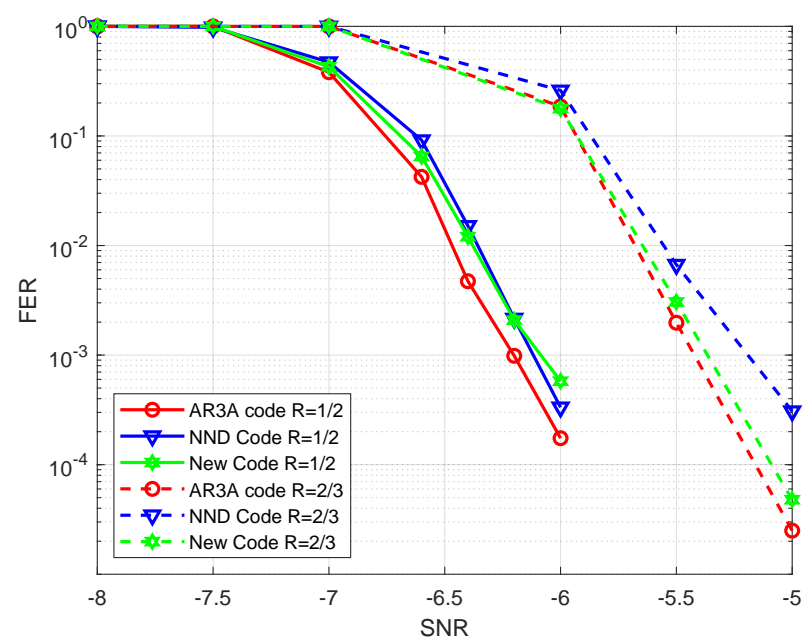

Figure 6. FER performance: Coding rate $R=1 / 2$ and $R=2 / 3$, information blocklength 2400 bits, 50 iterations, $10 \times 100$ LS-MIMO.

IEEE Communications Magazine, vol. 57, no. 8, pp. 84-90, Aug. 2019.

[3] Y. Fang, G. Bi, Y. L. Guan, and F. C. M. Lau, "A survey on protograph LDPC codes and their applications," IEEE Communications Surveys Tutorials, vol. 17, no. 4, pp. 19892016, 2015.

[4] Y. Fang, G. Han, G. Cai, F. C. Lau, P. Chen, and Y. L. Guan, "Design guidelines of low-density parity-check codes for magnetic recording systems," IEEE Communications Surveys $\mathcal{E}$ Tutorials, vol. 20, no. 2, pp. 1574-1606, 2018.

[5] Y. Fang, P. Chen, G. Cai, F. C. Lau, S. C. Liew, and G. Han, "Outage-limit-approaching channel coding for future wireless communications: Root-protograph lowdensity parity-check codes," IEEE Vehicular Technology Magazine, vol. 14, no. 2, pp. 85-93, 2019.

[6] F. Steiner, G. Böcherer, and G. Liva, "Protograph-based LDPC code design for shaped bit-metric decoding," IEEE Journal on Selected Areas in Communications, vol. 34, no. 2, pp. 397-407, 2015.

[7] C. Zhang, Y. Jing, Y. Huang, and X. You, "Massive MIMO with ternary ADCs," IEEE Signal Processing Letters, vol. 27, pp. 271-275, 2020.

[8] T. Liu, J. Tong, Q. Guo, J. Xi, Y. Yu, and Z. Xiao, "Energy efficiency of massive MIMO systems with low- 
resolution adcs and successive interference cancellation," IEEE Transactions on Wireless Communications, vol. 18, no. 8, pp. 3987-4002, Aug. 2019.

[9] J. Dai, J. Liu, J. Wang, J. Zhao, C. Cheng, and J. Wang, "Achievable rates for full-duplex massive MIMO systems with low-resolution ADCs/DACs," IEEE Access, vol. 7, pp. 24343-24353, 2019.

[10] S. Gao, P. Dong, Z. Pan, and G. Y. Li, “Deep learning based channel estimation for massive MIMO with mixed-resolution ADCs," IEEE Communications Letters, vol. 23, no. 11, pp. 1989-1993, Nov. 2019.

[11] L. V. Nguyen, D. T. Ngo, N. H. Tran, A. L. Swindlehurst, and D. H. N. Nguyen, "Supervised and semi-supervised learning for MIMO blind detection with low-resolution ADCs," IEEE Transactions on Wireless Communications, vol. 19, no. 4, pp. 2427-2442, 2020.

[12] L. Xu, X. Lu, S. Jin, F. Gao, and Y. Zhu, "On the uplink achievable rate of massive MIMO system with lowresolution ADC and RF impairments," IEEE Communications Letters, vol. 23, no. 3, pp. 502-505, Mar. 2019.

[13] T. V. Nguyen, H. D. Vu, D. N. Nguyen, and H. T. Nguyen, "Performance analysis of protograph LDPC codes over large-scale MIMO channels with low-resolution ADCs," IEEE Access, vol. 7, pp. 145145-145160, 2019.

[14] Y. Cho and S. Hong, "One-bit successive-cancellation soft-output (OSS) detector for uplink MU-MIMO systems with one-bit ADCs," IEEE Access, vol. 7, pp. 2717227182, 2019.

[15] F. Mousavi and A. Tadaion, "A simple two-stage detector for massive MIMO systems with one-bit ADCs," in Proceedings of the 27th Iranian Conf. Electrical Engineering (ICEE), Apr. 2019, pp. 1674-1678.

[16] Z. Shao, R. C. de Lamare, and L. T. Landau, "Iterative detection and decoding for large-scale multiple-antenna systems with 1-bit ADCs," IEEE Wireless Communications Letters, vol. 7, no. 3, pp. 476-479, 2017.

[17] H. Dang, T. Nguyen, and H. Nguyen, "Improve uplink achievable rate for massive MIMO systems with lowresolution ADCs," in Proceedings of the 20th IEEE Conference on Communications and Electronics (ICCE), Phu Quoc, Vietnam, Jan. 2021.

[18] H. N. Dang, T. Van Nguyen, and H. T. Nguyen, “On the performance of 1-bit adc in massive mimo communication systems," REV Journal on Electronics and Communications, vol. 10, no. 3-4, pp. 62-71, 2020.

[19] H. D. Vu, T. V. Nguyen, D. N. Nguyen, and H. T. Nguyen, "On design of protograph LDPC codes for large-scale MIMO systems," IEEE Access, vol. 8, pp. 46017-46029, 2020.

[20] D. Tse and P. Viswanath, Fundamentals Of Wireless Communication. Cambridge University Press, 2005.

[21] L. Fan, S. Jin, C. Wen, and H. Zhang, "Uplink achievable rate for massive MIMO systems with low-resolution ADC," IEEE Communications Letters, vol. 19, no. 12, pp. 2186-2189, Dec. 2015

[22] M. Srinivasan and S. Kalyani, "Analysis of massive MIMO with low-resolution ADC in Nakagami- $m$ fading," IEEE Communications Letters, vol. 23, no. 4, pp. 764767, Apr. 2019.

[23] W. Fukuda, T. Abiko, T. Nishimura, T. Ohgane, Y. Ogawa, Y. Ohwatari, and Y. Kishiyama, "Lowcomplexity detection based on belief propagation in a massive MIMO system," in Proceedings of the IEEE Vehicular Technology Conference (VTC), Jun. 2013, pp. 1-5.

[24] T. Takahashi, S. Ibi, and S. Sampei, "On normalization of matched filter belief in gabp for large MIMO detection," in Proceedings of the IEEE 84th Vehicular Technology Conference (VTC-Fall), Sep. 2016, pp. 1-6.

[25] T. V. Nguyen, A. Nosratinia, and D. Divsalar, "The design of rate-compatible protograph LDPC codes," IEEE Transactions on Communications, vol. 60 , no. 10 , pp. $2841-$ 2850, Oct. 2012.

[26] D. Divsalar, S. Dolinar, C. R. Jones, and K. Andrews,
"Capacity-approaching protograph codes," IEEE Journal on Selected Areas in Communications, vol. 27, no. 6, pp. 876-888, Aug. 2009.

[27] S. Abu-Surra, D. Divsalar, and W. E. Ryan, "On the existence of typical minimum distance for protographbased LDPC codes," in Proceedings of the Information Theory and Applications Workshop (ITA), Jan. 2010, pp. 1-7.

[28] Y. Fang, P. Chen, L. Wang, F. C. M. Lau, and K. K. Wong, "Performance analysis of protograph-based lowdensity parity-check codes with spatial diversity," IET Communications, vol. 6, no. 17, pp. 2941-2948, Nov. 2012.

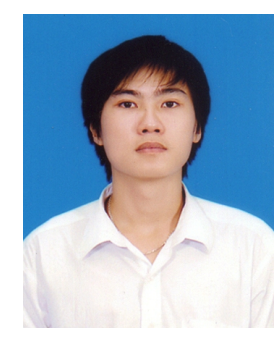

Hung N. Dang received the B.Sc. and M.Sc. degrees in information technology from Posts and Telecommunications Institute of Technology (PTIT), Hanoi, Vietnam. He is currently a lecturer of Faculty of Information Technology, Posts and Telecommunications Institute of Technology (PTIT), Hanoi, Vietnam. His research interests lie in Massive MIMO communications, channel coding design and analysis, wireless sensor networks.

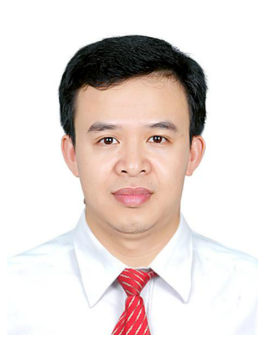

Thuy V. Nguyen received the B.Sc, M.Sc. and $\mathrm{Ph} . \mathrm{D}$. degrees in electrical engineering from Hanoi University of Science and Technology (HUST), Hanoi, Vietnam, New Mexico State University, Las Cruces, NM, USA, and the University of Texas at Dallas, Richardson, TX USA, respectively. He is currently a lecturer of Faculty of Information Technology, Posts and Telecommunications Institute of Technology (PTIT), Hanoi, Vietnam. Before joining PTIT, he was a Member of Technical Staff with Flash Channel Architecture, Seagate, Fremont, CA, USA. His research interests lie in the areas of coding theory and its applications in nextgeneration communication systems. 